\title{
Numerical Implementation and Comparison Between the Viscous, Fractional and Hysteretic Damping Models of a Rigid-Flexible Planar Manipulator
}

\author{
Jorge M. Martins, Alexandre N. Paris and José M. G. Sá da Costa
}

\begin{abstract}
This work treats the problem of modeling and identification of the structural damping of the flexible link of a rigid-flexible manipulator. Besides dissipating energy due to the joint friction, like in rigid robots, flexible robots also dissipate energy due to structural damping that results in the time amplitude decay of the flexible arms oscillations. This behavior is traditionally tackled recurring to viscous, fractional Voigt-Kelvin or hysteretic models. In this paper, for the latter two approaches, numerical approximations are used for their numerical implementation. Furthermore, a heuristic method based on the joint and flexible arm time responses is applied and compared with other methods, like recursive least-squares, to identify the large set of parameters of a flexible manipulator, including the inertial and joint friction parameters.
\end{abstract}

Index Terms - flexible manipulators, joint friction, structural damping, parameter identification.

\section{INTRODUCTION}

The importance of the dissipative components in the dynamic behavior of robot manipulators is such that any valid simulation model must include them. In the case of flexible robots there is energy dissipation due to the joints friction and energy dissipation due to internal friction in the flexible arms, generally known as structural damping.

The modeling of joints friction is complicated by the complexity of the phenomenon at low velocity and of the stick-slip process. Piedbœuf et al. [1] developed an algorithm that simulates the frictional behavior observed in experimental robot manipulators. This algorithm is based on a modification of the classical Coulomb model to include a stick-to-slip transition in a non-zero velocity necessary to prevent numerical problems. A standard static model is used to represent the friction in the slip phase while the stick phase is implemented as a joint-level constraint. Results of the identification and implementation of this friction model were presented in [2] and the model is further used in this work.

The majority of the bibliography dealing with the modeling of structural damping uses one of three models: the hysteretic model, the viscous and the fractional Voigt-Kelvin models. For the fractional model, Valério [3] describes a

This work was partly supported by POCTI/EME/41690/2001, Ministério da Ciência, Tecnologia e Ensino Superior, Fundação para a Ciência e a Tecnologia, Portugal.

The authors are with the Department of Mechanical Engineering and IDMEC at Instituto Superior Técnico, Technical University of Lisbon (TULisbon), Portugal \{martins, aparis, sadacosta\}@dem.ist.utl.pt fractional derivative obtained by transfer function approximation which we use in this work. The structural damping components are included in the dynamic equation of the model by applying the Virtual Power Principle.

The parameters identification for the inertial, the joint friction and the damping models, reveal to be quite difficult. The most used method for identification of rigid robots parameters is the recursive least-squares method [4] but it becomes complicated to find exciting trajectories when identifying the parameters of flexible manipulators. Dépincé [5] proposed an heuristic method to find the exiting trajectories based on identification by sets.

In this paper the recursive least-square method is used to identify only a set of the parameters that we are looking for. Other specific approaches are used to identify all the parameters including an heuristic approach that is developed based on the time response of the manipulator.

The paper is organized as follows: section 2 presents the dynamic model of the 2 joints flexible manipulator used in this work, section 3 describes the modeling of the structural damping components of a flexible beam. Section 4 describes the identification method implemented and presents the parameters identified. Validation results are presented in section 5 where the models are implemented in Matlab/Simulink to perform the simulation of a planar 2 joints flexible manipulator.

\section{DYNAMIC MODEL OF THE FLEXIBLE MANIPULATOR}

In this section we describe the manipulator dynamics equations [6]. For this work a two joints planar manipulator constituted by a rigid and a flexible link was used. A schematic representation of it is presented in Fig. 1. The flexible link is assumed as a Rayleigh beam clamped to the second joint, therefore with clamped-free boundary conditions. The dynamics equations were formulated using the Rayleigh-Ritz method and truncated in the third mode. The position of an infinitesimal element of the flexible link is obtained considering quadratic deformation.

The vector of generalized coordinates is defined as

$$
\boldsymbol{q}=\left[\begin{array}{lllll}
\theta_{1} & \theta_{2} & \eta_{1} & \eta_{2} & \eta_{3}
\end{array}\right]^{T}=\left[\begin{array}{ll}
\boldsymbol{q}_{\theta}^{T} & \boldsymbol{q}_{\eta}^{T}
\end{array}\right]^{T},
$$

where $\theta_{i}$ are the rigid coordinates and $\eta_{i}$ are the elastic coordinates defined as the time varying part of the RayleighRitz approximation of the bending deflexion. The dynamics equation of the flexible manipulator has the form

$$
\boldsymbol{M}(\boldsymbol{q}) \ddot{\boldsymbol{q}}+\boldsymbol{N}(\dot{\boldsymbol{q}}, \boldsymbol{q})+\boldsymbol{B}(\ddot{\boldsymbol{q}}, \dot{\boldsymbol{q}}, \boldsymbol{q})=\boldsymbol{\tau}
$$




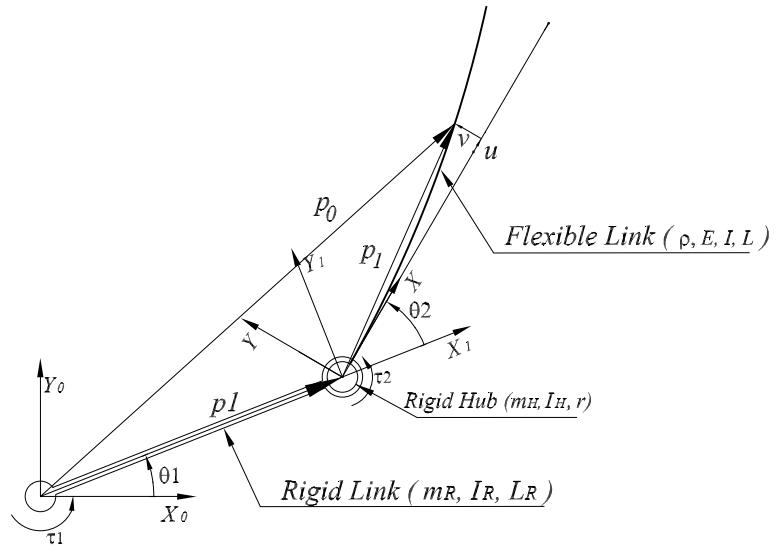

Fig. 1. Schematic representation of a planar 2 joint rigid-flexible manipulator

where $\boldsymbol{M}(\boldsymbol{q})$ is the inertial matrix of the system, $\boldsymbol{N}(\dot{\boldsymbol{q}}, \boldsymbol{q})$ is the vector of the stiffness, centrifugal and Coriolis forces. $\boldsymbol{B}(\ddot{\boldsymbol{q}}, \dot{\boldsymbol{q}}, \boldsymbol{q})$ is the vector of the dissipation forces of the system and $\tau$ is the vector of external forces.

\section{MODELING OF THE DISSIPATIVE COMPONENTS}

In this section we further describe the dissipation term of the dynamic equation of a flexible manipulator, $\boldsymbol{B}(\ddot{\boldsymbol{q}}, \dot{\boldsymbol{q}}, \boldsymbol{q})$. This term includes dissipation components due to the rigid part of the robot represented as $\boldsymbol{B}_{\theta}(\ddot{\boldsymbol{q}}, \dot{\boldsymbol{q}}, \boldsymbol{q})$ and components due to the flexible part represented as $\boldsymbol{B}_{\eta}\left(\dot{\boldsymbol{q}}_{\eta}, \boldsymbol{q}_{\eta}\right)$. The former is the friction in the joints of the robot, that was modeled and identified in [2] for this manipulator, and the latter is the damping in the flexible link characterized by an attenuation of the tip displacement in time when the link is in free vibration. Notice that $\ddot{\boldsymbol{q}}$ appears in $\boldsymbol{B}_{\theta}$ due to the modeling of stiction as a joint level constraint [1]. The dissipation vector has the form:

$$
\boldsymbol{B}(\ddot{\boldsymbol{q}}, \dot{\boldsymbol{q}}, \boldsymbol{q})=\left[\begin{array}{l}
\boldsymbol{B}_{\theta}(\ddot{\boldsymbol{q}}, \dot{\boldsymbol{q}}, \boldsymbol{q}) \\
\boldsymbol{B}_{\eta}\left(\dot{\boldsymbol{q}}_{\eta}, \boldsymbol{q}_{\eta}\right)
\end{array}\right] .
$$

\section{A. Structural damping}

1) Principle of Virtual Powers: To include the damping effect in the dynamic equation we apply the Principle of Virtual Powers or Jourdain's Principle to the fractional VoigtKelvin model. This model gives a good representation of systems with a low or high level of internal damping. Moreover, the equations developed for this model can be particularized to obtain the viscous damping model. The development is here described for the general 3D case of a Timoshenko beam and then particularized for the case study. Hooke's law for the fractional Voigt-Kelvin model is written as follows

$$
\begin{aligned}
& \sigma_{11}(t)=E \varepsilon_{11}(t)+E k_{e} D^{\alpha} \varepsilon_{11}(t) \\
& \tau_{12}(t)=G \gamma_{12}(t)+G k_{g} D^{\alpha} \gamma_{12}(t) \\
& \tau_{13}(t)=G \gamma_{13}(t)+G k_{g} D^{\alpha} \gamma_{13}(t)
\end{aligned}
$$

where the subscripts 1,2 and 3 represents, respectively, the $\mathrm{x}, \mathrm{y}$ and $\mathrm{z}$ frame directions on a beam cross section,
$E$ is the Young's modulus, $G$ is the shear modulus given by $G=E / 2$ and $k_{g, e}$ are the damping coefficients. The fractional derivative operator, also designated by noninteger order derivative, $D^{\alpha}(\cdot)$ is defined according to the RiemannLiouville definition as

$$
D^{\alpha} \varepsilon(t)=\frac{1}{\Gamma(1-\alpha)} \frac{d}{d t} \int_{0}^{t} \frac{\varepsilon(\tau)}{(t-\tau)^{\alpha}} d \tau
$$

where $\Gamma(\cdot)$ is the Gamma function and $\alpha$ is the order of the derivative that varies between zero and one. For $\alpha$ equal to one the fractional derivative becomes the first time-derivative and the Voigt-Kelvin model reduces to the classical VoigtKelvin model.

Writing (4) in matrix form yields

$$
\boldsymbol{\sigma}=\boldsymbol{E} \boldsymbol{G}+\boldsymbol{K}_{E} D^{\alpha} \boldsymbol{G}
$$

where $\boldsymbol{K}_{E}=\operatorname{diag}\left(E k_{e}, G k_{g}, G k_{g}\right), \boldsymbol{G}$ is the strain vector given by

$$
\boldsymbol{G}=\boldsymbol{\Gamma}+\widetilde{\boldsymbol{K}} \boldsymbol{Y}_{p} .
$$

$\Gamma$ represents the shear strains in the beam neutral axis:

$$
\boldsymbol{\Gamma}=\left[\begin{array}{lll}
\Gamma_{1} & \Gamma_{2} & \Gamma_{3}
\end{array}\right]^{T}=\left[\begin{array}{lll}
0 & \varphi_{12} & \varphi_{13}
\end{array}\right]^{T}
$$

with $\varphi_{12}$ and $\varphi_{13}$ being the shear angles as defined in the classical theory of elasticity. $\widetilde{\boldsymbol{K}}$ represents the skew symmetric matrix whose off-diagonal terms are the bending curvatures, i.e. $\boldsymbol{K}=\left[\begin{array}{lll}K_{1} & K_{2} & K_{3}\end{array}\right]^{T} . \boldsymbol{Y}_{p}=\left[\begin{array}{lll}0 & X_{2} & X_{3}\end{array}\right]^{T}$ is the position of a material point $p$ of the beam in a certain cross section, relative to the origin of the cross section reference frame.

The virtual power of the internal elastic stress defined in (6) is stated as follows

$$
\int_{\mathcal{B}} \delta \dot{\boldsymbol{G}}^{T} \boldsymbol{\sigma} d \mathcal{B}=\int_{\mathcal{B}} \delta \dot{\boldsymbol{G}}^{T}\left(\boldsymbol{E} \boldsymbol{G}+\boldsymbol{K}_{E} D^{\alpha} \boldsymbol{G}\right) d \mathcal{B}
$$

where $\mathcal{B}$ represents the body of the beam in the undeformed configuration.

Considering that the beam cross sections are symmetric and remain plane after deformation, making some matrix manipulations and assuming a Rayleigh-Ritz expansion of the elastic variables, the elastic dissipative term reduces to [7]

$$
\boldsymbol{B}_{\eta}\left(D^{\alpha} \overline{\bar{q}_{\eta}}\right)=\int_{0}^{L}\left[\begin{array}{cc}
\mathcal{K}_{K} & 0 \\
0 & \mathcal{K}_{\gamma}
\end{array}\right] d X_{1} D^{\alpha} \overline{\boldsymbol{q}_{\eta}} .
$$

with $X_{1}$ being the position of a cross section along the beam neutral fiber,

$$
\mathcal{K}_{K}=\left[\begin{array}{cc}
E k_{e} I_{3} \boldsymbol{v}_{2 x}^{\prime \prime} \boldsymbol{v}_{2 x}^{\prime \prime}{ }^{T} & 0 \\
0 & E k_{e} I_{2} \boldsymbol{v}_{3 x}^{\prime \prime} \boldsymbol{v}_{3 x}^{\prime \prime}{ }^{T}
\end{array}\right]
$$

and

$\mathcal{K}_{\gamma}=\left[\begin{array}{ccc}G k_{g} J \boldsymbol{\alpha}_{x}^{\prime} \boldsymbol{\alpha}_{x}^{\prime T} & 0 & 0 \\ 0 & G k_{g} A \boldsymbol{\gamma}_{2 x}^{\prime} \boldsymbol{\gamma}_{2 x}^{\prime}{ }^{T} & 0 \\ 0 & 0 & G k_{g} A \boldsymbol{\gamma}_{3 x}^{\prime} \boldsymbol{\gamma}_{3 x}^{\prime}{ }^{T}\end{array}\right]$

where $\boldsymbol{v}_{2 x}, \boldsymbol{v}_{3 x}, \boldsymbol{\alpha}_{x}, \gamma_{2 x}$ and $\gamma_{3 x}$ are vectors of shape functions for the elastic deflections and $\overline{\boldsymbol{q}_{\eta}}=$ 
$\left[\boldsymbol{v}_{2 t} \boldsymbol{v}_{3 t} \boldsymbol{\alpha}_{t} \gamma_{2 t} \gamma_{3 t}\right]^{T}$ is the vector of the generalized elastic coordinates [8].

Equation (10) is valid for the general Timoshenko 3D case. For our case study where we have a 2D Rayleigh flexible beam, described in section II, (10) is simplified as follows: the planar configuration implies that $v_{3 t}=0$; due to the flexible beam geometry the torsion in the $x$ axis vanishes resulting in $\alpha_{t}=0$; and neglecting shear strain, $\gamma_{2 t}=\gamma_{3 t}=$ 0 . The elastic coordinate vector reduces to the matrix block element that defines the beam deflection relative to the $y$ axis, and (10) can be written as

$$
\boldsymbol{B}_{\eta}\left(D^{\alpha} \boldsymbol{q}_{\eta}\right)=\int_{0}^{L}\left[E k_{e} I_{3} \boldsymbol{v}_{2 x}^{\prime \prime} \boldsymbol{v}_{2 x}^{\prime \prime}\right] d X_{1} D^{\alpha} \boldsymbol{q}_{\eta}
$$

where $\boldsymbol{v}_{2 t}=\boldsymbol{q}_{\eta}=\left[\begin{array}{lll}\eta_{1} & \eta_{2} \eta_{3}\end{array}\right]^{T}$.

2) Viscous damping: The viscous damping is defined as a function of the first derivative of the generalized elastic coordinates, i.e. when $\alpha$ equals to one. Equation (13) is rewritten as

$$
\boldsymbol{B}_{\eta}\left(\dot{\boldsymbol{q}}_{\eta}\right)=\int_{0}^{L}\left[E k_{e} I_{3} \boldsymbol{v}_{2 x}^{\prime \prime} \boldsymbol{v}_{2 x}^{\prime \prime T}\right] d X_{1} \dot{\boldsymbol{q}}_{\eta}
$$

3) Fractional damping: The fractional model gives a better approximation to the real behavior of the damping at low and high frequencies. This model is obtained considering a value for the order of the derivative $\alpha$ between zero and one in (13). It is then necessary to calculate the fractional derivative. One of the ways to calculate the derivative is through (5). This expression is difficult to deal with mathematically because it yields a very complex numerical implementation. Another option for the calculation of the fractional derivative is through an approximation in the frequency domain given in [3] as

$$
G(s)=s^{\alpha} .
$$

The Bode diagram of this function has a slope between 0 and $20 \mathrm{~dB} / \mathrm{dec}$ in the gain curve. The approximation is made considering a high order integer transfer function, with a behavior close enough to the one desired, but much easier to handle.

4) Hysteretic damping: The constant hysteretic damping model is often used to describe the dynamic behavior of structures undergoing various loading conditions. In order to introduce this model, textbooks usually start with the more conventional viscous damping model for a single-degree-offreedom system

$$
m \ddot{x}+c \dot{x}+k x=f(t)
$$

where $m, c$ and $k$ represent the mass, viscous damping coefficient and stiffness, respectively, and $f(t)$ the exciting force. This equation is an ordinary second order differential equation with constant coefficients. When excited by a harmonic force at frequency $\omega$, it can be proven that for each vibration cycle the system dissipates, through its viscous damper, a quantity of energy directly proportional to the damping coefficient, the excitation frequency and the square of the response amplitude, i.e.,

$$
W_{\text {diss. }}=\int_{0}^{2 \pi / \omega} f \dot{x} d t=\pi c \omega X^{2}
$$

that is the area of the ellipse defined by the forcedisplacement plot. However, experimental results from tests performed on a large variety of materials show that damping due to internal friction is nearly independent of the forcing frequency but still proportional to the square of the response amplitude, i.e.,

$$
W_{\text {diss. }} \simeq \alpha X^{2} \text {. }
$$

Therefore, from (17) and (18) an equivalent viscous damping coefficient may be defined as

$$
c_{e q}=\frac{\alpha}{\pi \omega}=\frac{h}{\omega}
$$

and the equivalent damping force given by $\frac{h}{\omega} \dot{x}$. In such conditions, (16) becomes the equilibrium equation of a single-degree-of-freedom system with hysteretic damping formulated as [9]

$$
m \ddot{x}+\frac{h}{\omega} \dot{x}+k x=F e^{i \omega t}
$$

where $h$ is the hysteretic damping coefficient and $F$ and $\omega$ are the amplitude and exciting frequency of the harmonic load, respectively. Making $\dot{x}=i \omega x$, we can rewrite (20) in the form [10]

$$
m \ddot{x}+k\left(1+i \eta_{H}\right) x=F e^{i \omega t}
$$

where $\eta_{H}=h / k$ is the hysteretic damping ratio. Although good for harmonic motion, (21) is invalid for free vibration since, when the excitation force, $F e^{i \omega t}$, doesn't exist, the presence of $\omega$ in the denominator of (20) does not make any physical sense. Therefore, only the steady-state solution can be obtained. In other words, the hysteretic damping is focused on the frequency domain model as follows [11]

$$
-m \omega^{2} x+k\left(1+\operatorname{sign}(\omega) i \eta_{H}\right) x=F
$$

where $\operatorname{sign}(\omega)$ is +1 when $\omega>0$ and -1 when $\omega<0$. The time-domain approach was obtained by taking the inverse Fourier transform of (22) resulting in

$$
m \ddot{x}(t)-\frac{k \eta_{H}}{\pi} \int_{-\infty}^{\infty} \frac{x(\tau)}{(t-\tau)} d \tau+k x(t)=F(t)
$$

with the conditions at $t=-\infty$ being

$$
\left.x(t)\right|_{t=-\infty}=0,\left.\quad \dot{x}(t)\right|_{t=-\infty}=0
$$

If the excitation load $F(t)$ in (23) vanishes, the solution $x(t)$ becomes the free vibration response of the system.

The problem with (23) is its numerical implementation. Since it is a non-causal equation, its solution must be obtained iteratively [11], which is not compatible with a simulation environment such as Simulink. An alternative approach is to use a describing function, such as in the work of Symens et al. [12] where the free vibration response is obtained by substituting the hysteretic term in (22) by a 


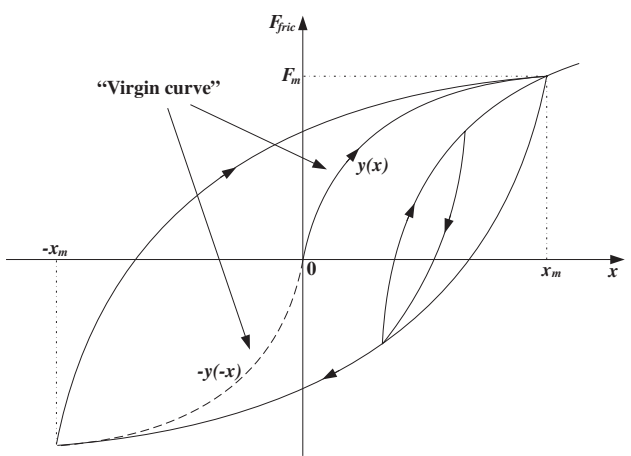

Fig. 2. Example of a hysteresis trajectory.

displacement depended function that represents the hysteretic trajectory. This function depends on the previous history of the movement. Thus, referring to Fig. 2, if there has never been a relative movement before, the friction force follows a virgin curve $f(x)$

$$
\begin{aligned}
& F_{\text {fric }}=f(x) \\
& \text { with }
\end{aligned}
$$

If the motion direction changes at $x=x_{m}$ the friction force becomes:

$$
\begin{array}{cc}
F_{\text {fric }} & =F_{m}+2 f\left(\frac{x-x_{m}}{2}\right) \\
\text { with } & F_{m}=F_{\text {fric }}\left(x_{m}\right)
\end{array}
$$

If the movement reverses again, before $x=x_{m}$ the same reversal rule (26) is used for the force. If, on the contrary, the direction of the movement at $x=-x_{m}$ has not reversed, friction force follows rule (25) again. A reasonable and analytically tractable form for the "virgin curve", derived from experiments is

$$
y=h_{0}\left[1-\left(\frac{\delta}{\delta+x}\right)^{2}\right]
$$

with $h_{0}$ and $\delta$ being characteristic scale factors for force and displacement.

\section{IDENTIFICATION}

After determining the model equation we have now to identify its parameters. The parameters are here divided into two sets described as follow:

Inertial and stiffness model parameters: the rotation inertia of link 1 and the inertia of joint 2 relative to joint 1 axis of rotation, $I_{R 0}+m_{H} L_{r}^{2}$; rotation inertia of joint 2 relative to its center of mass, $I_{H}$; the cross section mass of the flexible link, $\rho A$; the cross section moment of inertia of the flexible link, $\rho I_{3}$; the elastic stiffness of the flexible link, $E I_{3}$.

Structural damping model parameters: damping coefficient for the viscous model, $k_{e}$; damping coefficient and fractional derivative order for the fractional model, $k_{e}$ and $\alpha$, respectively; damping coefficient $k_{e}$, and the characteristic scale factors $h_{0}$ and $\delta$ of the hysteretic model.

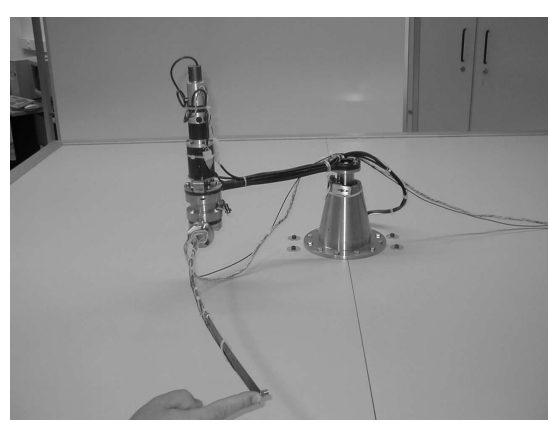

Fig. 3. Experimental 2 joint planar rigid-flexible robot manipulator

\section{A. Identification of the dynamic model parameters}

The inertial and stiffness parameters can still be divided in rigid parameters, $I_{R 0}+m_{H} L_{r}^{2}$ and $I_{H}$, and elastic parameters, $\rho A, \rho I_{3}$ and $E I_{3}$. The a priori numerical values of these parameters, determined by geometrical measures and material characteristics, are given in Table I. These parameters are not the real ones because they do not include the mass of the cables and sensors installed in the robot (see Fig. 3), nor the motors rotation inertia.

\section{TABLE I}

A priori VALUES OF THE DYNAMICAL PARAMETERS

\begin{tabular}{lllll}
\hline $\begin{array}{l}I_{R 0}+m_{H} L_{r}^{2} \\
{\left[\mathrm{~kg} . \mathrm{m}^{2}\right]}\end{array}$ & $I_{H}$ & $\rho A$ & $\rho I_{3}$ & $E I_{3}$ \\
\hline 0.298 & $\left.13 . \mathrm{m}^{2}\right]$ & {$[\mathrm{kg} / \mathrm{m}]$} & {$[\mathrm{kg} . \mathrm{m}]$} & {$\left[\mathrm{Nm}^{2}\right]$} \\
\hline
\end{tabular}

1) Recursive least-squares method: The least-squares algorithm based on the dynamic model of the system (2) is the most used method to identify the standard parameters of a model. To make the process of identification faster and more efficient computationally, we apply the recursive least-squares (RLS) method that permits the online identification [4]. The model is linear with respect to the following standard set of parameters: $I_{R 0}+m_{H} L_{r}^{2}, I_{H}, \rho A, \rho I_{3}$ and $E I_{3}$. [5] proposed some heuristic methods that allow us to find the exciting trajectories to identify parameters of flexible robots. One of the methods consists in applying an input torque in the frequencies of the first two modes of the flexible arm, but experimental results show that, due to the coupling in our system, we have to find a combination of the two joint torques that permits to identify the rigid and elastic parameters simultaneously. To verify the significance of the identified parameters we calculate the relative contribution of each parameters to the total force of the system, given by [5]

$$
C r(i)=\frac{\left\|H_{i} \theta_{i}\right\|}{\sum\left\|H_{i} \theta_{i}\right\|}
$$

where $H_{i}$ is the column $i$ of the regression matrix and $\theta_{i}$ is the component $i$ of the parameter vector $\left(\theta_{i}\right.$ should not be confused here with the joint coordinates). The regression matrix is obtained by differentiating (2) with respect to the standard parameters.

The identified parameters through RLS are in Table II where we show the error relative to the a priori parameters, 
$e_{a p}$. The results presented in Table II show that the contribution of the parameter $\rho I_{3}$ is not significant, and therefore the flexible link may be assumed as an Euler-Bernoulli beam. Furthermore, for this system, it is shown in [2] that joint friction torque represents more than $50 \%$ of the total joint torque.

TABLE II

DYNAMIC PARAMETERS ESTIMATED BY RLS

\begin{tabular}{lllll}
\hline & $\hat{\theta}_{a p}$ & $\hat{\theta}$ & $\mathrm{Cr}(\%)$ & $e_{a p}(\%)$ \\
\hline$I_{R 0}+m_{H} L_{r}^{2}$ & 0.298 & 0.3428 & 24.33 & 15.00 \\
$I_{H}$ & $13.22 \mathrm{E}-4$ & 0.0297 & 6.85 & 2148.6 \\
$\rho A$ & 0.1570 & 0.1891 & 8.95 & 20.45 \\
$\rho I_{3}$ & $1.31 \mathrm{E}-8$ & $1.77 \mathrm{E}-4$ & 0.13 & $1.36 \mathrm{E} 6$ \\
$E I_{3}$ & 0.3490 & 0.3677 & 6.82 & 5.36 \\
\hline
\end{tabular}

2) Identification of the elastic inertial parameters through static deflections: Even if results of the RLS identification are correct, we apply other identification methods as a way of comparison. The identification of the elastic parameters $E I_{3}$ and $\rho A$, can be done by measuring the static deflection, $\nu$, of the flexible beam due to applied loads, $F$, at the free end. With this data, $E I_{3}$ and $\rho A$ can be identified by linear regression from the following equation [13]

$$
\nu=\frac{L^{3}}{E I_{3}}\left(\frac{F}{3}-\frac{\rho A g L}{8}\right)
$$

where $L$ is the length of the flexible beam. The results for the identification of the elastic parameters are presented in Table III.

TABLE III

ESTIMATED ELASTIC PARAMETERS BY STATIC DEFLECTIONS.

\begin{tabular}{llll}
\hline & $\hat{\theta}_{a p}$ & $\hat{\theta}$ & $e_{a p}(\%)$ \\
\hline$\rho A$ & 0.1570 & 0.1685 & 7.3 \\
$E I_{3}$ & 0.3490 & 0.3122 & 10.5 \\
\hline
\end{tabular}

3) Identification of the rigid inertial parameters with heuristic method: The heuristic method consist in change the parameters values in the simulation model to minimize the quadratic error between the model response and the previously acquired real robot response to a same torque input. To do this we use the function for constrained minimization fmincon, of MATLAB. The function to be minimized accepts a parameter vector generated by the fmincon, simulates the model with this parameters and returns the quadratic error between the model and real response in each iteration. The fmincon upper limits was defined after various tested values, with lower limits close to zero and initial values based on the a priori values. The tolerances was very low to achieve the better approximation. To make easy the method-name connection this method is hereafter designated as fmincon optimization. With this method we can identify the rigid dynamic parameters, $I_{R 0}+m_{H} L_{r}^{2}$ and $I_{H}$ (presented in Table IV) and the structural damping parameters, $\alpha, k_{e}$, $h_{0}$ and $\delta$ (section IV-B). The fmincon optimization doesn't achieve good results when trying to obtain the rigid and elastic parameters at the same time.
TABLE IV

fmincon ESTIMATED RIGID PARAMETERS

\begin{tabular}{llll}
\hline & $\hat{\theta}_{a p}$ & $\hat{\theta}$ & $e_{a p}(\%)$ \\
\hline$I_{R 0}+m_{H} L_{r}^{2}$ & 0.298 & 0.3894 & 30.7 \\
$I_{H}$ & $13.22 \mathrm{E}-4$ & 0.0268 & 1927.2 \\
\hline
\end{tabular}

\section{B. Identification of the damping parameters}

For the identification of the damping parameters we apply a method proposed in [13] based on the beam frequency response. The damping coefficients $k_{e}$, and the fractional derivative order $\alpha$ are identified using the following expression [13]:

$$
\zeta+k_{e} \omega^{\alpha}\left(\zeta \cos \left(\frac{\pi \alpha}{2}\right)-\sin \left(\frac{\pi \alpha}{2}\right)\right)=0
$$

where $\omega$ is the natural frequency associated with each beam mode and $\zeta$ is the modal damping parameter. We use the information of the first two modes to obtain the damping coefficient $k_{e}$ and the fractional derivative order $\alpha$. The two equations resulting from using (30) are squared, summed and minimized for the variables $k_{e}$ and $\alpha$ using the function fminsearch of MATLAB. For the viscous damping model, the order of the derivative is 1 . Thus, we get from (30)

$$
\zeta-k_{e} \omega=0
$$

and $k_{e}$ is readily isolated using the information from the first mode.

We can also use the fmincon function to identify the damping parameters. The cost function in this case is the mean square error between the tip displacement obtained experimentally and the one obtained by simulation. The tip displacement obtained by simulation uses information of the elastic parameters that were obtained by two methods, RLS and static deflections. Experimental results show that the frequency response is better when using elastic parameters obtained by static deflection. Table $\mathrm{V}$ show the results for $k_{e}$ and $\alpha$ obtained from the modes parameters and using fmincon function, and the hysteretic parameters obtained by fmincon.

TABLE V

STRUCTURAL DAMPING MODEL PARAMETERS.

\begin{tabular}{llllll}
\hline \multicolumn{3}{c}{ Modes parameters } & \multicolumn{3}{l}{ fmincon optimization } \\
\hline & Viscous & Fractional & Viscous & Fractional & Hysteretic \\
\hline$k_{e}$ & $3.46 \mathrm{E}-4$ & 0.010 & $7.04 \mathrm{E}-4$ & 0.028 & 0.020 \\
$\alpha$ & - & 0.218 & - & 0.185 & - \\
$h_{0}$ & - & - & - & - & 0.189 \\
$\delta$ & - & - & - & - & $4.884 \mathrm{E}-4$ \\
\hline
\end{tabular}

\section{VALIDATION}

The results presented are obtained with the rigid inertial parameters, the structural damping parameters and the viscous friction parameters identified by fmincon optimization and the elastic inertial parameters identified by RLS. Fig. 4 show the power spectrum of the real robot comparing it with the three models, where we can see that the viscous model 
just capture the first two vibration modes being the worst damping model.

Fig. 5 compares the trajectory followed by the real robot to the model trajectory with the same controller. Fig. 6 shows the tip displacement where the increasing in time of the error is due to the error accumulated during the calculation of the displacement in the model.
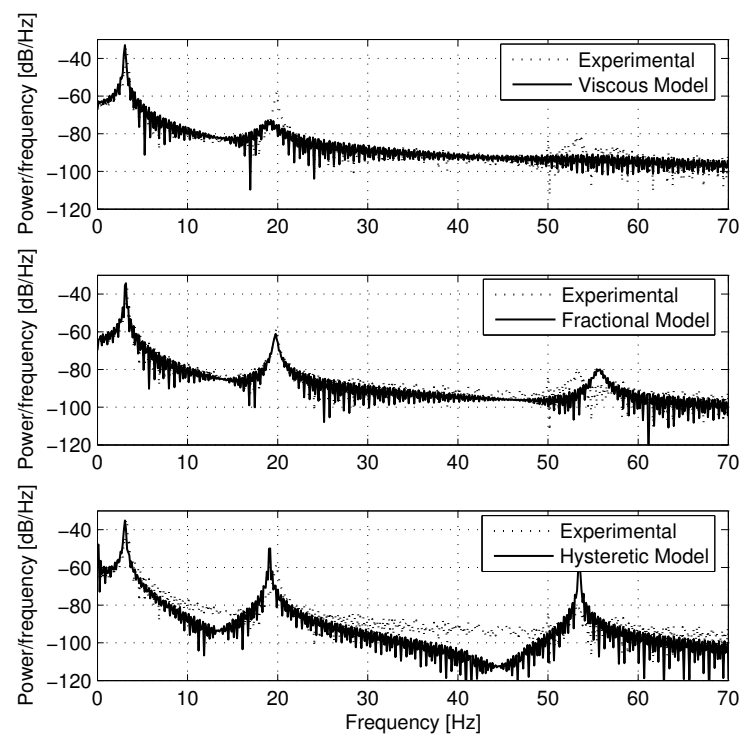

Fig. 4. Spectrum.
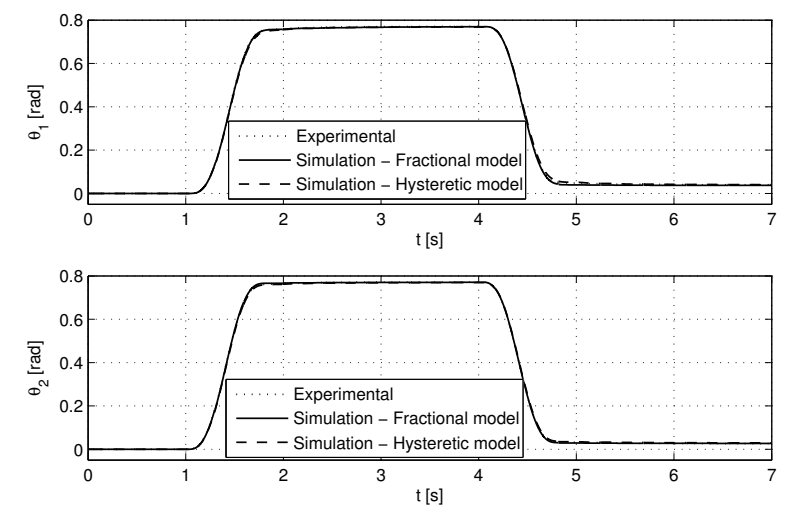

Fig. 5. Joint trajectory.

\section{CONCLUSIONS}

In order to obtain a robot model with a behavior similar to the real robot, this research performs a compilation of the methods used for formulation and parameters identification of a 2 joints flexible robot model that includes the structural damping in the flexible link. For the modeling of the structural damping the hysteretic model reveals to be more accurate than the viscous and fractional model in both frequency and time response. In terms of identification, the elastic parameters identified through static deflections achieve a more accurate frequency response than the ones identified by RLS method. The last one obtains better results

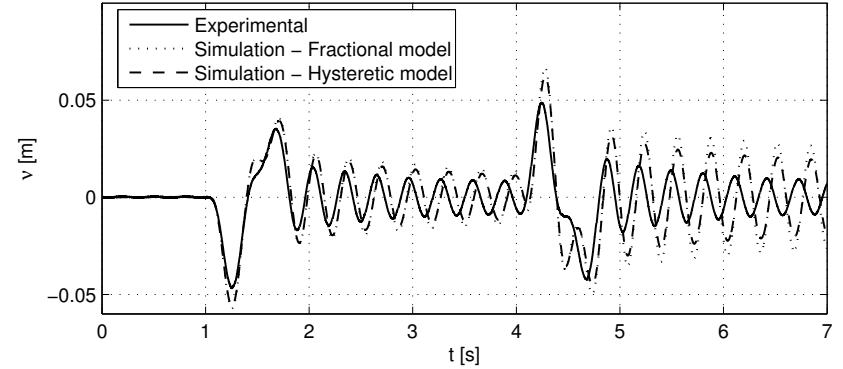

Fig. 6. Tip displacement

for the time response. The damping parameters obtained by the heuristic method produce better results than the ones obtained analytically. Therefore, the results reveal that one method is not better than the other in every situation.

\section{ACKNOWLEDGEMENTS}

The authors would like to thank Prof. Relógio Ribeiro and Dr. Duarte Valério for their help and availability to clarify many questions.

\section{REFERENCES}

[1] J.-C. Piedbœuf, J. De Carufel, and R. Hurteau, "Friction and stickslip in robots: Simulation and experimentation," Multibody System Dynamic, no. 4, pp. 341-354, 2000.

[2] A. N. Paris, J. M. Martins, and J. S. da Costa, "On the modeling of structural damping and dynamic parameter identification of a rigid-flexible planar manipulator," in 7th Portuguese Conference On Automatic Control, Lisboa, Portugal, Sept.11-13, 2006.

[3] D. Valério and J. S. da Costa, "Time-domain implementation of fractional order controllers," IEE Proc.-Control Theory Appl., vol. 152, no. 5, pp. 539-552, September 2005.

[4] G. Calafiore, M. Indri, and B. Bona, "Robot dynamic calibration: Optimal excitation trajectories and experimental parameter estimation," Journal of Robotic Systems, vol. 18, no. 2, pp. 55-68, 2001.

[5] P. Dépincé, "Parameters identification of flexible robots," Proceedings of the 1998 IEEE International Conference on Robotics \& Automation, pp. 1116-1121, May 1998.

[6] J. Martins, M. A. Botto, and J. S. da Costa, "Modeling of flexible beams for robotic manipulators," Multibody System Dynamics, vol. 7, pp. 79-100, 2002.

[7] J. Martins, J. Sá da Costa, and M. Botto, "Modelling, Control and Validation of Flexible Robot Manipulators," in Advances in Computaional Multibody Systems, ser. Computational Methods in Applied Sciences, J. A. Ambrósio, Ed. Dordrecht, The Netherlands: Springer, 2005, pp. 239-268.

[8] J. Martins, J. S. da Costa, and M. A. Botto, "Modeling, control and validation of flexible robot manipulators," in Advances in Computational Multibody Systems, J. A. C. Ambrósio, Ed. Springer-Verlag, 2005, pp. 239-268.

[9] L. Meirovitch, Elements of vibration analysis, 2nd ed. New York: McGraw-Hill, 1986.

[10] A. M. R. Ribeiro, N. M. M. Maia, J. Silva, L. Reis, and M. Freitas, "Free vibration response using the constant hysteretic damping model." Timisoara, Romania: The $11^{\text {th }}$ International Conference on Vibration Engineering, September 27-30 2005.

[11] J. T. Chen and D. W. You, "Hysteretic damping revisited," Advances in Engineering Software, vol. 28, no. 3, pp. 165-171, April 1997.

[12] W. Symens, F. Al-Bender, J. Swevers, and H. V. Brussel, "Dynamic characterization of hysteresis elements in mechanical systems," Proceedings of the American Control Conference, pp. 4129-4134, May 2002.

[13] J.-C. Piedbøuf, L.-L. Pagé, I. Tremblay, and M.-J. Potvin, "Efficient simulation of a multilayer viscoelastic beam using an equivalent homogeneous beam," Proceedings of the 1999 IEEE International Conference on Robotics \& Automation, pp. 1188-1193, May 1999. 